\title{
Are computers of any use in a family planning clinic?
}

\author{
Alison Bigrigg, FRCS (Ed), MRCOG, DM MFFP, MBA \\ Clinical Directorate, Family Planning and Sexual Health Directorate, Glasgow, Scotland, UK
}

(Accepted September $9^{\text {th }}, 1999$ )

\section{Key words}

audit management; family planning clinics; information technology

\section{Key message points \\ - Information technology can facilitate efficient clinic administration and appointment systems in the family planning clinic. \\ - Electronic client records can be used in family planning clinics, but it is difficult to gain agreement from clinicians as to what data to collect. \\ - Electronic client records can facilitate clinical audit. \\ - Useful information technology in a family planning clinic is viewed positively by clients.}

\section{Introduction}

Four years ago, at the end of the financial year 1994/95, the Family Planning Directorate in Glasgow received trust financing to purchase computer hardware and software to update the family planning appointment system at the central clinic, and to introduce electronic clinical records for clients attending that clinic. It was expected that this would improve the efficiency of the clinic administration and raise standards in the following four clinical areas:

- clinic management

- clinical communication

- clinical audit

- clinical information.

This paper discusses current use of information technology (IT) within the clinic and considers how far these objectives have been achieved.

\section{The Glasgow information technology system}

The Glasgow Family Planning and Sexual Health Directorate operates from one central clinic (2 Claremont Terrace) and 30 peripheral sites. Outreach work is also undertaken in client's homes, youth clubs, hostels for the homeless and similar sites.

At the central clinic the appointment system is fully computerised, while at the peripheral clinics a traditional appointment book is used. At the central clinic clinicians enter clinical information directly onto the computers during the consultation or immediately afterwards. Traditional written records are also maintained for any information which is not on the computerised programme. In the peripheral clinics traditional written notes are the clinical records, and a 'statistics' sheet is kept for management purpose. This records 10 items of basic information for statistical returns to the Trust and Scottish Office.

At the central clinic the clinicians are able to record most of the information required for a consultation regarding contraception onto the computer directly (Figures 1a and 1b). The data set for well-woman consultations has not proved so easy to develop, and written notes are more extensively used. For most contraceptive consultations no written notes are required, but clinicians may choose to note points of interest such as 'going on honeymoon to Florida' or aspects of counselling which have been specially emphasised.

Figure 1a Information recorded directly onto computer during consultations regarding contraception at Glasgow Central Family Planning Clinic. Choice of computer programme available to clinician

$$
\begin{array}{ll}
\text { 1. } & \text { Contraception } \\
\text { - } & \text { initial assessment } \\
\text { - } & \text { follow-up visit } \\
\text { - } & \text { counselling only }
\end{array}
$$

\section{Emergency contraception}

3. Laboratory tests only

4. Prescription only

5. Specialist services colposcopy medical gynaecology psychosexual counselling ultrasound scanning

lesbian health

vasectomy

medical gynaecology

HPV

erectile dysfunction

Base 75 (for prostitutes)

breast awareness

6. General health information/well woman screen

Clinicians also record on the computer when they take a cervical smear or swab, and results are entered into the computer when they return from the laboratory (electronic transfer may be possible in the future). Results are then available on screen when the patient returns. Standard letters informing clients and GPs of the test results are generated on a daily basis.

It is clinic policy to inform GPs of initial prescriptions of contraception and when there is a change of method. These letters can also be generated immediately at the end of the consultation.

Outreach and specialist services, such as vasectomy and colposcopy, also use the system to record patient information directly, but these functions will not be described in this paper.

The family planning computer system is not linked to other primary care trust computer systems, and no access is allowed to staff outside the Directorate. Within the Directorate, each member of staff has a password and code number. Access to different levels of information is dependent on need. Administrative staff cannot access clinical information using their code number. 
Figure 1b Information recorded directly onto computer during consultations regarding contraception at Glasgow Central Family Planning Clinic. Initial consultation for contraception

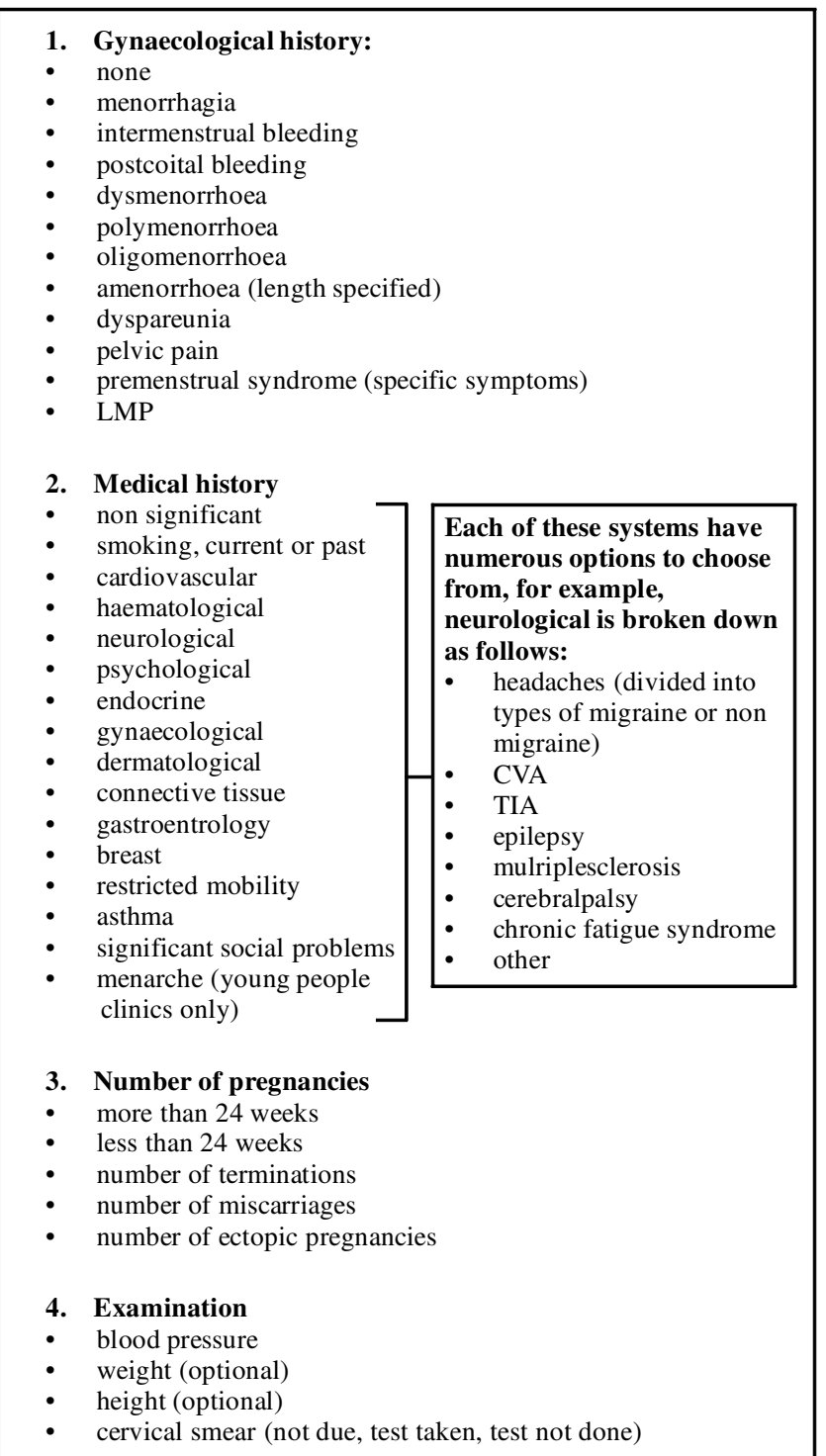

\section{Current method of contraception}

6. Any sexual difficulties: YES/NO

7. Family history Each category is broken down to give further details, for example, breast

- cardiovascular $-\quad<50$ years old in first degree relative

- breast cancer $\quad>50$ years in first degree relative breast cancer $\_$- or strong family history

8. Current medication

- none

- anti-epileptiform

- antibiotics

- anticoagulants

- antifungal

- antihypertensives

- antidepressants

- antidiabetes

- other

9. Contraception prescribed

- type

- amount, e.g. number of packets

- other details, e.g. pelvic examination and coil insertion

10. Staff present at consultation
Figure 1c Information recorded directly onto computer during consultations regarding contraception at Glasgow Central Family Planning Clinic. Follow up consultation for contraception

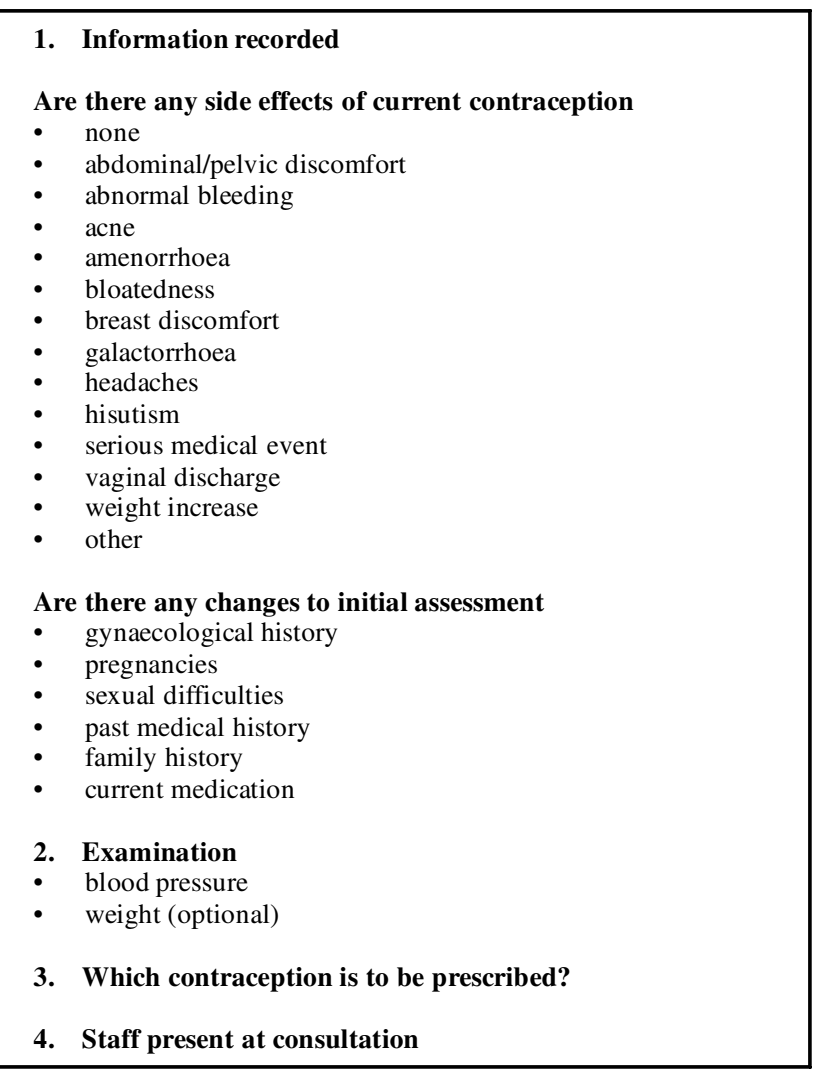

The central clinic had two word processors in 1994; there are now 33 distributed around the clinic as shown in Figure 2. All the computers are linked to each other and to the server.

For better or worse, the Directorate chose to purchase a software package which had to be 'customised' to the clinic. The advantage is that changes can be made to the database whenever a clinician wishes. The disadvantages are that database construction is more complex. All these functions were not achieved at once, and Figure 3 shows a timetable by which the Directorate has developed IT.

Reports from the system are produced by a clerical office (A\&C Grade 4) who is employed on a full-time basis to do this, train personnel and maintain the equipment.

The software for the system in Glasgow is known as DP4. It is produced and supported by In-Practice Systems (formally Reuters Health Information). The majority of the computer terminals are AST Bravo and the server is an Elonex NT 3.5. The system contains information on over 100000 individuals who have attended the peripheral or central clinic.

\section{Client viewpoint}

In 1997 a questionnaire was given to 300 clients inquiring about their views on the use of computers in the central clinic. Of these 185 were regular attenders, and 115 were new clients. A quarter of the sample were teenagers and a tenth were over 36 years old.

The majority thought that computers were necessary and were not more time consuming during the clinical consultation. In addition, most clients thought enough care was taken with confidentiality of their personal records and they were happy with the personal details held on file about them.

In many ways, however, the comments in a 'free comment' area on the questionnaire were most illuminating. 
Figure 2 Computer hardware in Glasgow Central Family Planning Clinic.

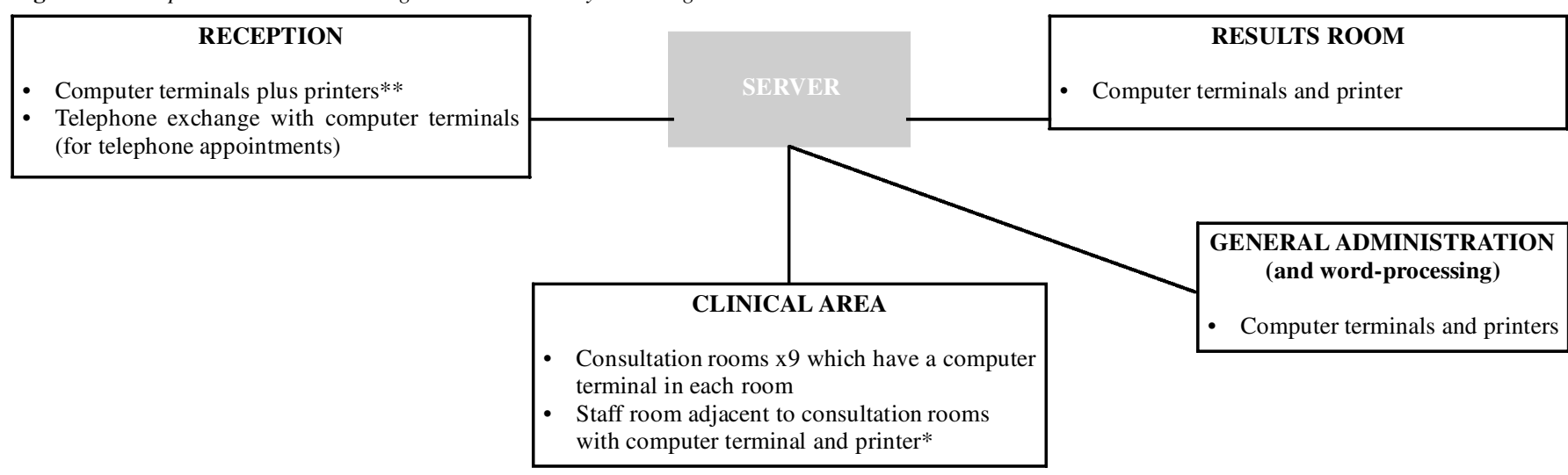

*This printer is directly linked to the computers in the consulting rooms. It will print patient labels and all standard letters.
**This printer mainly produces labels. For notes and lists of patients whose notes are required from records.
The vast majority of comments were positive; some examples are shown below:

"I wasn't really aware of computers until I filled this in. I find it heart warming that an organisation is bothering to find out peoples reactions to computers in this day and age"

"Introduction of computer database can only improve service. Very efficient and friendly. Will definitely use it again"

"Always generally concerned that information on computer databases is over simplified to fit in with parameters of the database and therefore not reflecting everything absolutely accurately"

"Have been attending for over 20 years and the service is better, warmer and friendlier than ever"

\section{How well has the system met the initial objectives? \\ Patient administration}

The computerised appointment system replaced 18 different appointment books. Now the clerical staff cannot imagine working without computers. Processes such as finding the next available appointment, finding an appointment when the client has forgotten when it is, changing the times of clinics, ensuring accuracy of patient demographic details and 'pulling notes' for the clinics are greatly simplified. However, as the role of IT in patient administration is well established, this subject will not be described in any further detail.

\section{Clinic management}

The ability to produce timely and accurate information has

Figure 3 Timetable for introduction of IT:

\begin{tabular}{ll}
\hline March 1994 & Equipment and software purchased \\
\hline October 1995 & Computerised appointment system goes 'live' \\
\hline January 1996 & $\begin{array}{l}\text { Clinicians enter limited amount of data required for } \\
\text { Scottish Office reports during consultation (4 items) }\end{array}$ \\
\hline January 1997 & $\begin{array}{l}\text { Complete electronic records for contraception } \\
\text { introduced }\end{array}$ \\
\hline July 1997 & $\begin{array}{l}\text { Database for contraception modified and simplified to } \\
\text { that shown in Figure 2. }\end{array}$ \\
\hline
\end{tabular}

saved time for administrative staff in producing statutory reports. It has also meant that clinical managers are able to monitor workload and staffing more accurately.

A regular report is produced giving average number of attendances for each clinic session, and the average number of attendances per member of staff. In the central clinic the workload can be broken down by type of consultation, for example, postcoital contraception or request for termination of pregnancy. Staffing levels are adjusted appropriately. Another report shows the number of contraceptive caps and IUD's fitted on average in each clinic session. This can be used by trainees to locate desired training opportunities.

As long as individual doctors or nurses have entered their personal code at the beginning of each consultation, they can request a list of IUD's they have fitted or cervical smears they have taken in a specified time period. This information is used for personal re-accreditation purposes.

The appointment system went 'live' 2 weeks before the Pill scare of 1995 (which was a source of some logistical problems). If a similar Pill scare occurred now, the clinic would be able to identify every client to whom it had supplied a specific type of pill and take appropriate action.

When one of our peripheral sites was closed due to the loss of the building lease, we were able to inform every client registered with the clinic individually (as long as they gave a correspondence address) of where alternative services could be accessed.

Information on waiting times can also be analysed. This information has been rather horrifying to clinical managers, but at least we are aware of the extent of the problem and are taking some measures to tackle it.

\section{Clinical communication}

The system allows for GPs to receive a clear, wordprocessed, letter summarising the family planning clinic consultation. This replaces previous handwritten letters with obvious benefits. The greatest improvement in communication, however, has been the standard letters automatically generated to clients and GPs with cervical smear and swab results. The system automatically blocks the printing of letters to clients or general practitioners if permission for correspondence has not been given.

The electronic records of cervical smears are also useful in a busy clinic when 'chasing the paper copy' can be time consuming, especially when it may have been filed three or four years ago and the client cannot remember exactly when a repeat smear is due. 
If a client has attended a peripheral clinic, the IT system will currently only give limited information about that consultation if they subsequently attend the central clinic. In the future, closer liaison and electronic linking to laboratories and GP systems should produce much greater improvement in quality of services. Other possibilities of the system which have not yet been exploited include the generation of personalised information leaflets, as well as direct linking of the clinical programme to reference information for staff.

\section{Clinical audit / clinical governance}

The information system will never be suitable for all aspects of clinical audit. In particular, patient views and clinician attitudes require other techniques. It is, however, being used in Glasgow to monitor simple outcome measures such as:

- percentage of unsatisfactory smears

- number of smears taken in teenagers

- percentage of positive Chlamydia swabs

- percentage of consultations involving COC supply where blood pressure was not recorded

- number of Nova T's inserted

- number of clients referred for termination of pregnancy following previous attendance in our service

- number of clients re-attending with problems relating to IUD insertion.

The IT system produces figures which usually require further investigation, but it is useful to screen for problems and focus investigation on appropriate client groups.

Information can be obtained for the clinic as a whole, each clinic session or individual clinicians. In addition, the system assists audit by identifying groups of clients, for example, those who have had hysteroscopy in the last year. They can then be sent a questionnaire concerning patient satisfaction, or other relevant information.

\section{Clinical information}

To date, the clinical information contained within the system has not been fully utilised. Only regular, simple, reports are produced, for example, methods of contraception prescribed (Figure 4). In the future it is intended to produce, and report in peer reviewed journals, such information as the contraceptive choices of clients with specific medical conditions such as epilepsy or obesity, or actual side effects experience by different client types with specific methods.

Information available from the peripheral sites is more limited, but each clinic is supplied with a synopsis of its activity on an annual basis, with more specific reports produced on request. The output is, of course, limited by the data put in.

Figure 4a Breakdown by contraception given

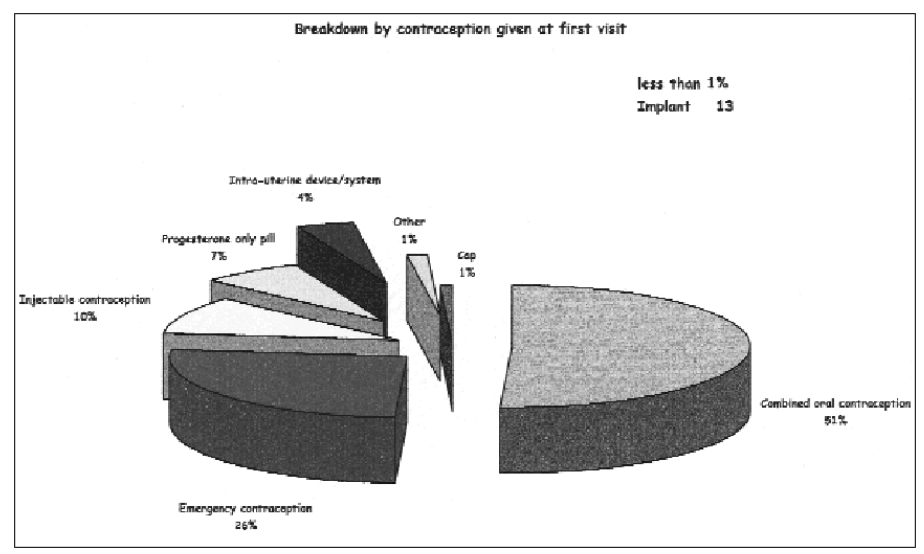

Figure 4b Breakdown by $C O C$

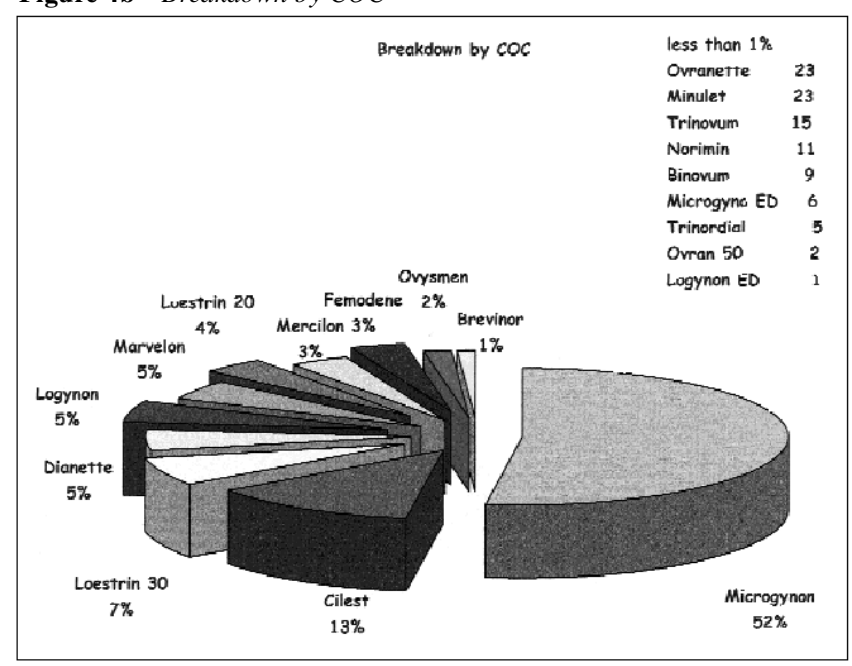

\section{Discussion}

The system is now embedded in the culture of the central clinic. Many of the advantages are taken for granted, and disadvantages overlooked. Intuitively it is felt that the system improves the standard of record keeping, and probably increases the consultation time. Regrettably, neither of these beliefs have ever been investigated. A more in depth look at client preferences should also be undertaken, but impressions from the User Group and other sources of client views remain favourable.

With such a large clinic, continuous staff turnover is inevitable. No one has been unable to master the computer function so far, and indeed some staff members who have never used computers in the past, have been encouraged to do so in other aspects of their lives. Initially our staff who had never used computers were offered a half day course, 'Computers for the uninitiated', which was provided by a local training company. In addition, senior staff and administrative staff with advanced knowledge were on hand for 1-2 weeks at the clinic, whenever a new aspect of the system was introduced. Subsequently, new staff have been trained how to use the computers during their induction session, when a senior doctor or nurse explains the clinics systems, health and safety, and other clinic regulations.

Undoubtedly some aspects of the database are clumsy, and the technology is already looking out-dated. Much of this relates to our inexperience in designing databases and lack of expert help. Reports from the system are now available, but it has taken much time and grief to achieve what the suppliers said would be easy. Much information held in the system still remains unused.

The Glasgow experience will not be transferable to every family planning clinic. The size of service, geographical location and staff expertise all vary from one area to another and effect local requirements for information.

\section{Conclusion}

The IT system provides support to many aspects of administration and clinical care. If asked whether the same decision to computerise the clinic would be taken if there were the chance to start again, the answer would undoubtedly be 'yes', but the clinic would benefit from hindsight when choosing a system and method of implementation.

Statements on funding and competing interests

Funding. None.

Competing interests. None. 